\title{
COLECISTECTOMIA LAPAROSCÓPICA COM ABORDAGEM SUPRA-PÚBICA
}

\author{
Laparoscopic cholecystectomy with suprapubic approach
}

\author{
Marcus Vinícius Andrade Chalar SILVA, Dionísio Ferreira de ALMEIDA, Maxley Martins ALVES, \\ Marco Aurélio Gonçalves de Andrade BARBOSA, Miro Walter Carneiro VIEIRA
}

Trabalho realizado no serviço de Cirurgia Geral do Hospital Garavelo, Aparecida de Goiânia, Goiás, Brasil.

DESCRITORES - Colelitíase. Laparoscopia. Colecistectomia laparoscópica. Técnicas.
RESUMO - Racional: As colecistectomias laparoscópicas realizadas através de acesso abdominal único, apesar de seu inquestionável resultado estético, são procedimentos de custo elevado e tecnicamente difíceis de serem realizados. A abordagem suprapúbica é alternativa mais simples e barata com bom resultado estético. Objetivo: Relatar a experiência do Hospital Garavelo com a colecistectomia laparoscópica com abordagem supra-púbica. Método: Estudo descritivo de delineamento transversal e coleta retrospectiva dos dados. Foram avaliados o tempo cirúrgico, taxas de sucesso e de conversão para procedimento laparoscópico tradicional ou mesmo operação aberta, bem como a ocorrência de complicações intra ou pós-operatórias e tempo de internação. Os dados foram coletados através de instrumento criado especificamente para realização do estudo, permitindo a coleta de dados quantitativos dos registros hospitalares e observações feitas durante avaliações ambulatoriais. Resultados: A amostra foi constituída por 42 pacientes. O sexo feminino foi predominante $(76,2 \%)$. A média de idade foi de 36 anos (18-65). A taxa de sucesso foi de 95,3\%. O tempo médio para realização do procedimento foi de 33,4 minutos. Não houve complicações intra-operatórias e todos os pacientes receberam alta hospitalar nas primeiras 24 horas após a operação. Conclusão: A colecistectomia laparoscópica com abordagem supra-púbica é técnica segura, de fácil domínio, com bom resultado estético, podendo ser técnica alternativa para os procedimentos convencionais.

\section{Correspondência: \\ Marcus Vinícius Andrade Chalar Silva \\ E-mail : marcuschalar@hotmail.com \\ Fonte de financiamento: não há \\ Conflito de interesses: não há \\ Recebido para publicação: 21/02/2013 Aceito para publicação: 21/05/2013}

HEADINGS - Cholelithiasis. Laparoscopy. Laparoscopic cholecystectomy.Techniques.
ABSTRACT - Background: The laparoscopic cholecystectomies performed through single site surgery, despite undoubted aesthetic results, are costly and technically difficult to be performed. The suprapubic approach presents as a simpler and cheaper alternative with good aesthetic results. Aim: To report the experience of Garavelo Hospital on laparoscopic cholecystectomy with suprapubic approach. Methods: Descriptive, crossectional study with retrospective data retrieval. The variables to be analyzed were success rate; occurrence of complications; surgical time and the length of stay. Results: The sample consisted of 42 patients, of which females were predominant (76.2\%). The age ranged from 18 to 65 years with an average age of 36 years. The success rate was $95.3 \%$. The average time for the procedure was 33.4 minutes. There were no intraoperative complications and all patients were discharged within 24 hours after surgery. Conclusion: Laparoscopic cholecystectomy with suprapubic approach is safe and easy to domain. It can be performed in a time similar to traditional laparoscopy, without special instruments. It offers a good cosmetic result, and deserves more attention.

\section{INTRODUÇÃO}

utilização da via supra-púbica como alternativa para o
posicionamento de portais laparoscópicos não é recente. Em
1995 Degano et al ${ }^{7}$ propuseram sua utilização para realização de colecistectomias. Apesar de seu relato inicial ter sido realizado a 17 anos, muito pouco foi publicado sobre seu uso desde então. Tal fato decorreu em parte devido ao surgimento e grande interesse despertado pelas operações laparoscópicas com acesso abdominal único em diversos países, inclusive no Brasil 1,13,14. Hoje, grande número de doenças podem ser tratadas através deste tipo de técnica; entretanto, o elevado custo dos materiais e as dificuldades inerentes a esse tipo de procedimento dificultam sua aplicação como método 
de rotina ${ }^{4,6}$.

Atualmente pode-se observar ressurgimento de publicações utilizando a abordagem supra-púbica em todo o mundo $3,5,8-12,15,16$. Como este tipo de operação pode ser realizada com o mesmo material necessário para realização das laparoscopias convencionais, dispensando uso de materiais descartáveis, seu custo é significativamente inferior ao das operações por acesso abdominal único. Este fato associado à relativa facilidade de execução faz dessa técnica alternativa que pode ser mais bem explorada.

O presente estudo tem por objetivo relatar a experiência do Hospital Garavelo com a colecistectomia laparoscópica através da abordagem supra-púbica.

\section{MÉTODOS}

Trata-se de estudo descritivo de delineamento transversal e coleta retrospectiva dos dados. Foi avaliado e aprovado pelo Comitê de Ética em Pesquisa número CAAE 01351812.6.0000.0033 da instituição.

O estudo foi realizado no serviço de Cirurgia Geral do Hospital Garavelo em Aparecida de Goiânia, Goiás em parceria com o programa de residência médica em Cirurgia Geral do Hospital Santa Genoveva de Goiânia, $\mathrm{GO}$, Brasil. A população constituiu-se de pacientes com indicação para realização de colecistectomia eletiva devido a colelitíase sintomática, oriundos tanto da clínica privada, quanto encaminhados pela Secretaria da Saúde através de sua central de regulação, no período entre 2011 e 2012. Nesse período foram realizados 42 colecistectomia laparoscópicas em que a abordagem supra-púbica foi utilizada.

Os dados foram coletados através de instrumento criado especificamente para realização do estudo, permitindo a coleta de dados quantitativos dos registros hospitalares e observações feitas durante avaliações ambulatoriais. A análise dos dados foi realizada utilizando software EPI-INFO. Devido ao tamanho reduzido da amostra foi utilizado o teste exato de Fisher para determinação de significância estatística.

\section{Técnica operatória}

O paciente é posicionado em decúbito dorsal com o braço direito paralelo ao corpo de modo idêntico à colecistectomia laparoscópica tradicional. Com relação à posição do cirurgião e dos auxiliares a totalidade dos procedimentos foi realizado em posição americana com o cirurgião e o auxiliar situados ao lado esquerdo do paciente.

Nos casos iniciais foi posicionada sonda vesical de demora com retirada ainda no centro cirúrgico, após o término do procedimento; porém, este procedimento foi posteriormente dispensado, sendo solicitado ao paciente que urinasse antes do encaminhamento para o centro cirúrgico. Não foram observadas dificuldades adicionais nos casos subsequentes.
O pneumoperitônio foi realizado com agulha de Veress naqueles sem intervenção cirúrgica prévia e por técnica de Hasson naqueles pacientes com manipulação cirúrgica no abdômen ou pelve; a insuflação de cavidade abdominal foi realizada até pressão de $15 \mathrm{mmHg}$.

$O$ acesso inicial foi realizado com trocarte de $12 \mathrm{~mm}$ em cicatriz umbilical para passagem de ótica de $5 \mathrm{~mm}$. Após avaliação inicial era determinado se a operação seria ou não factível com abordagem suprapúbica. Em caso afirmativo eram posicionados dois trocárteres de $5 \mathrm{~mm}$ em região supra-púbica na borda lateral do músculo reto abdominal direito e esquerdo (Figura 1)

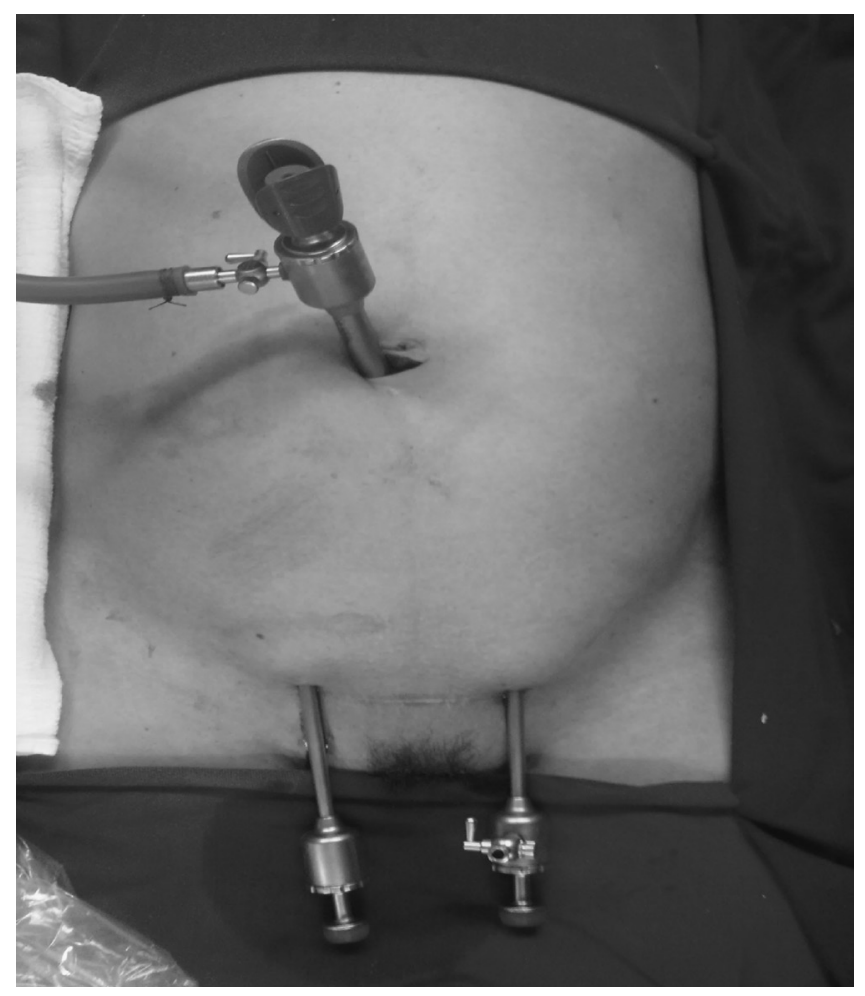

FIGURA 1 - Posicionamento dos portais na operação com abordagem supra-púbica

Neste momento a ótica era deslocada para o trocarte de $5 \mathrm{~mm}$ localizado lateralmente ao músculo reto abdominal esquerdo e realizada aproximação com recurso zoom. Pelo trocarte de $5 \mathrm{~mm}$ da direita era posicionada pinça para apreensão do infundíbulo e pelo trocarte umbilical as pinças para dissecção, tesoura e clipador de 5 ou $10 \mathrm{~mm}$, conforme o caso. A visão conseguida para dissecção do pedículo da vesícula biliar era praticamente a mesma da laparoscopia tradicional, não aumentando assim os riscos do procedimento (Figura 2 ). 


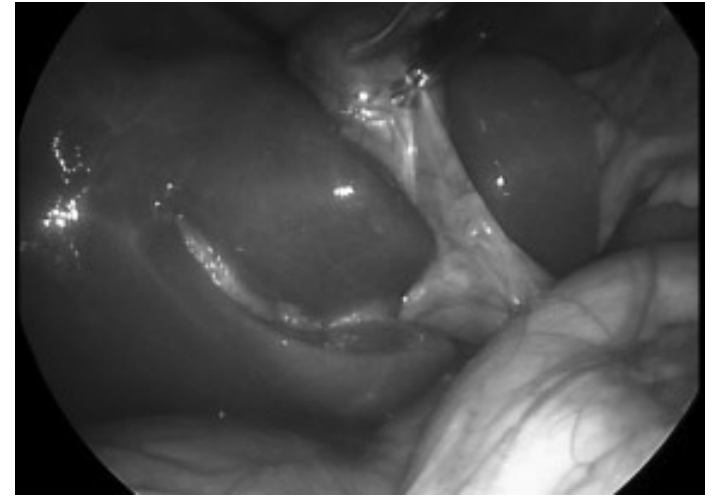

FIGURA 2 - Visão através do portal supra-púbico esquerdo

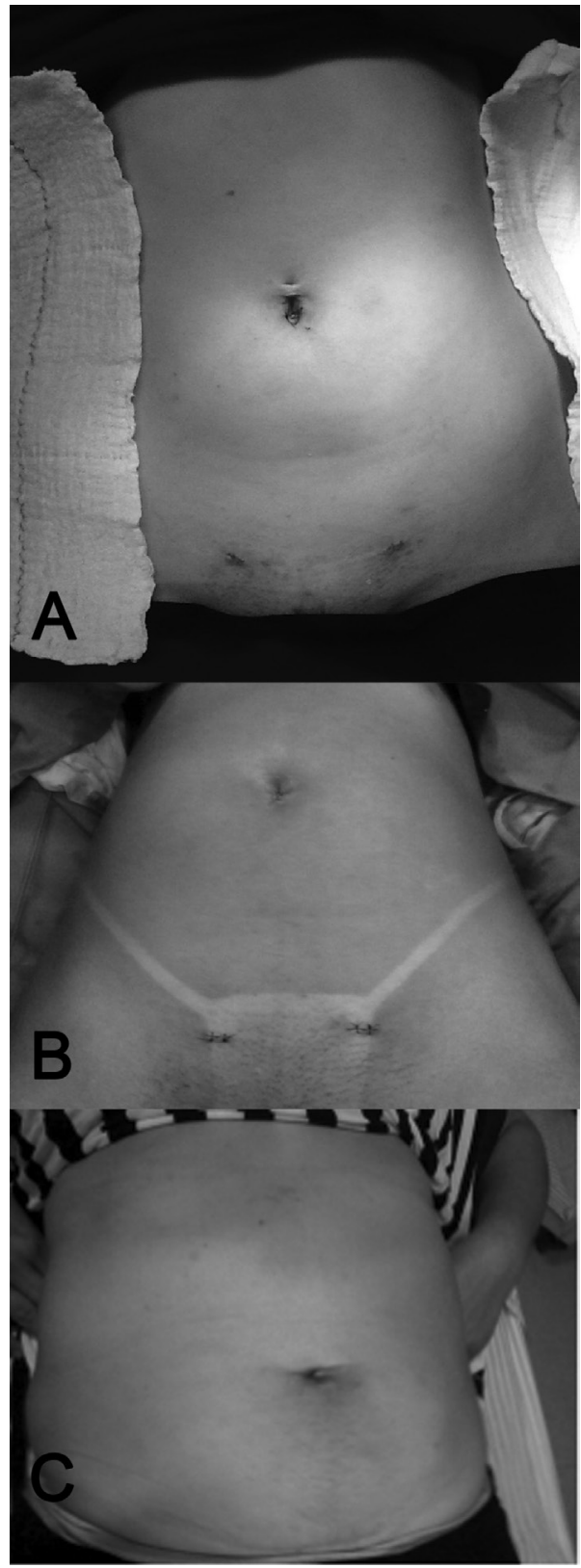

FIGURA 3 - Aspecto pós-operatório imediato: A) paciente com parto cesáreo prévio; B) sem incisão prévia; C) em 30 dias de pós-operatório
Nos casos iniciais, ou naqueles em que foi observada dificuldade na exposição segura do pedículo da vesícula biliar foi utilizado portal adicional de 3 $\mathrm{mm}$ em região do flanco direito entre as linhas axilar anterior e média para tração do fundo da vesícula de forma semelhante ao que ocorre na laparoscopia tradicional.

O restante da operação transcorria de modo idêntico à laparoscópica tradicional com a peça cirúrgica retirada em bolsa protetora pela cicatriz umbilical. O defeito aponeurótico na cicatriz umbilical era fechado; nos demais, era realizada apenas a síntese do defeito cutâneo (Figura 3).

Foram avaliados o tempo cirúrgico, taxas de sucesso e de conversão para procedimento laparoscópico tradicional ou mesmo operação aberta, bem como a ocorrência de complicações intra ou pósoperatórias e tempo de internação.

RESULTADOS

Foram realizados 42 procedimentos pela técnica supra-púbica. O grupo de pacientes era composto em sua maioria por mulheres que correspondiam a 76,2\%; os homens correspondiam a $23,8 \%$. Com relação à idade a média deste grupo foi de 36 anos variando entre 18 e 65 anos. Os pacientes provenientes da clínica privada representaram $47,6 \%$ e os provenientes da rede pública $52,4 \%$.

Nesta série, o procedimento foi concluído com êxito em 95,3\% dos casos. Em um paciente foi necessário uso de um portal adicional de $5 \mathrm{~mm}$ em hipocôndrio direito e em outro conversão para procedimento laparoscópico tradicional. O uso do portal adicional de $3 \mathrm{~mm}$ no flanco direito ocorreu em 31\%, sendo mais frequentemente utilizado nos primeiros casos. O tempo médio para realização do procedimento foi de 33,4 (DP 7,95 minutos). Todos receberam alta hospitalar nas primeiras 24 horas do pós-operatório. Na primeira revisão cirúrgica, realizada entre sete e 14 dias, observou-se a presença de coleção subcutânea em três pacientes. Não foram identificados fatores que os diferenciassem dos demais; todos tiveram boa resposta com aspiração por agulha. Na revisão do $30^{\circ}$ dia do pós-operatório nenhum dos pacientes referia dor em sitio cirúrgico que impactasse de forma negativa suas atividades diárias.

\section{DISCUSSÃO}

O primeiro relato da utilização da via supra-púbica para realização de colecistectomias laparoscópicas foi na Itália em 1995 por Degano et al. ${ }^{7}$ No decorrer dos anos foram propostas modificações relacionadas ao número e diâmetro dos portais e seu posicionamento. A disposição utilizada nesta série foi a de Leggett e seu grupo em $2001^{12}$. Uma questão importante para realização deste tipo de procedimento consiste no 
equipamento de laparoscopia disponível na unidade e sua capacidade de fornecer imagens em formato digital com possibilidade de aproximação de imagem. A ferramenta zoom, apesar de ser de grande valia, não é essencial em todos os casos. Em pacientes com biotipo favorável pode-se conseguir o mesmo efeito aproximando a ótica da área de dissecção. Neste contexto é importante notar que a proximidade da ótica e do portal umbilical, que neste momento estão dispostos praticamente em paralelo, pode levar a choques constantes entre o cirurgião e o assistente. Esse tipo de dificuldade é idêntica a que ocorre durante as operações laparoscópicas realizadas através de acesso abdominal único ${ }^{1,6}$, exigindo maior experiência da equipe com este outro tipo de procedimento.

Até o momento desta publicação, foram identificados dez trabalhos nos quais foi utilizada a via supra-púbica para colecistectomia. Destas, nove foram publicadas em periódicos ${ }^{5,7-12}$ e uma apresentada sob a forma de pôster em reunião da Society of Gastrointestinal and Endoscopic Surgeons $(\mathrm{SAGES})^{16}$. Além destas, existe referência ao uso deste procedimento em uma página pessoal ${ }^{15}$, onde são disponibilizadas imagens dos procedimentos. A análise dos dados e das imagens permite observar que se trata de um procedimento semelhante ao realizado por Legget et al ${ }^{12}$; porém, o cirurgião assistente mantinha o uso do portal epigástrico, como descrito originalmente por Degano et al. ${ }^{7}$ Nesta pesquisa não conseguiu-se identificar se esses dados foram publicados; porém, segundo dados da próprio site, o grupo realiza este procedimento desde 2004. Como o autor dessa página figura entre os autores do trabalho apresentado por Spindel et al. ${ }^{16} \mathrm{e}$ em virtude da escassez de material disponível optou-se por incluí-la nesta discussão.

Analisando séries publicadas, é possível contabilizar que do momento da descrição há 17 anos atrás até o período atual, foram realizadas 275 colecistectomias com uso da via supra-púbica. Nesses 275 casos a taxa de conversão foi de $1,04 \%(n=2)$, o que demonstra a relativa facilidade de adaptação do cirurgião e de sua equipe. Com relação à taxa de conversão uma observação deve ser feita. Como não existe padronização deste tipo de procedimento, observa-se ampla variação no modo com que os diversos grupos realizam o procedimento. Degano et al. ${ }^{12}$ na série original e Spindel et al. ${ }^{16}$, cujas séries somadas totalizam 113 pacientes, ou seja, 41\% da amostra, já iniciam o procedimento com o uso de um portal subxifoidiano, o que nas demais séries, inclusive nesta que se relata, já seria considerado conversão para procedimento tradicional. A adoção deste posicionamento se baseia no fato de que, ao utilizar um portal na parte superior da parede abdominal anterior, determina-se prejuízo estético, comprometendo assim a única vantagem realmente comprovada deste tipo de procedimento. Os autores de forma idêntica àqueles que avaliam a cirurgia laparoscópica através de acesso abdominal único, fazem referência à melhor recuperação funcional; porém, tal fato ainda não foi comprovado. Pode-se esperar que o uso da via suprapúbica, quando comparada ao acesso abdominal único, resulte sim em menor dor pós-operatória e menor índice de complicações relacionadas à ferida cirúrgica pela menor tensão gerada na parede abdominal por este tipo de procedimento ${ }^{2}$.

A maioria dos casos reportados nas diversas séries consiste de pacientes previamente selecionados, envolvendo apenas casos simples, muitas vezes oligossintomáticos e com perfil físico favorável. Assim, são necessários mais estudos para definir as reais possibilidades desta variante técnica.

Segundo os dados reportados pelos diversos autores as operações foram realizadas com tempo médio de 39,86 minutos, não sendo reportadas complicações pós-operatórias. Os únicos relatos de conversões para laparoscopia tradicional vem da série publicada por Degano et al ${ }^{1}$ onde foi necessária em $6,8 \%$ dos casos. Com relação à evolução, a maioria dos pacientes recebeu alta com menos de 24 horas; porém, existem relatados casos em que foi necessária internação prolongada atingindo até cinco dias, não sendo fornecida explicação para este fato pelos autores 11 .

De modo geral os dados obtidos neste estudo não diferem dos observados na literatura. A taxa de conversão para laparoscopia tradicional neste estudo foi de $4,7 \%(n=2)$, acima da reportada de $1,04 \%$, sem significância estatística $(p=0,15)$. Convém recordar de que a definição de conversão tem significados diferentes para os diversos autores. O tempo cirúrgico médio observado foi de 33,4 minutos, abaixo dos reportados de 40 minutos. Apesar de estatisticamente significativa $(p<0,0001)$ essa diferença tem pouco impacto na prática clínica diária.

Não houve complicações cirúrgicas nesta série, tal qual referido na literatura disponível. Nas revisões pós-operatórias foi observada presença de coleção subcutânea em $7 \%(\mathrm{~N}=3)$ dos pacientes, todos com boa evolução após aspiração.

Apesar da necessidade de três punções abdominais, os portais inferiores situam-se em áreas habitualmente não expostas, podendo também ser posicionados sobre cicatrizes de operações ginecológicas/obstétricas prévias. O tempo necessário para realização dos procedimentos não é muito diferente do necessário para realização de colecistectomia laparoscópica tradicional. A taxa de complicações foi nula nesta série bem como na literatura disponível. Além disso, ao preservar minimamente a triangulação entre os portais, a técnica com posicionamento alternativo torna mais fácil a adaptação do cirurgião e sua equipe. A questão econômica também deve ser levada em conta, pois todo o procedimento pode ser realizado com o material cirúrgico permanente utilizado em laparoscopia tradicional. 


\section{CONCLUSÃO}

A colecistectomia laparoscópica com abordagem supra-púbica é técnica segura e de fácil domínio. Pode ser realizada em tempo semelhante ao da laparoscopia tradicional e não necessita de acessórios especiais, o que contribui para menor custo final. Apresenta bom resultado estético e boa recuperação funcional.

\section{REFERÊNCIAS}

1. Antoniou SA, Pointner $R$, Granderath FA. Single-incision laparoscopic cholecystectomy: a systematic review. Surg Endosc. 2011 Feb;25(2):367-77.

2. Blinman $T$ - Incisions do not simply sum: Surg.Endosc. 2010;24(7):1746-5

3. Cala Z, Niksić K, Nesek-Adam V, Klapan D, Soldo I. Cosmetic laparoscopic cholecystectomy. J Laparoendosc Adv Surg Tech A. 2006 Dec;16(6):577-81.

4. Connor S. Single-Port-Access Cholecystectomy: History Should Not Be Allowed to Repeat World J Surg. 2009;33:1020-1021

5. Cruz-Munoz N, Koniaris L. Alternative port site selection (APSS) for improved cosmesis in laparoscopic surgery. J Gastrointest Surg. 2010 Dec;14(12):2004-8.

6. Curcillo PG 2nd, Wu AS, Podolsky ER, Graybeal C, Katkhouda N, Saenz A, Dunham R, Fendley S, Neff M, Copper C, Bessler M, Gumbs AA, Norton M, Iannelli A, Mason R, Moazzez A, Cohen L, Mouhlas A, Poor A. Single-port-access (SPA) cholecystectomy: a multi-institutional report of the first 297 cases. Surg Endosc. 2010 Aug;24(8):1854-60.
7. Degano G, Santarelli E, Ceraudo E, Iantosca F, Taccaliti F, Monaco M. Video-laparoscopic cholecystectomy with suprapubic approach. Technical note Minerva Chir. 1995 Dec;50(12):1109-14.

8. Federmann GF, Hessler $\mathrm{CH}$. Hidden laparoscopic access (HiLA) cholecystectomy - first results. Eur Surg. 2011;43(1):34-38.

9. Ersoz F, Ozcan O, Sari S, Bektas H, Arikan S. Laparoscopic cholecystectomy on the bikini line for invisible scar. Surg Laparosc Endosc Percutan Tech. 2011 Feb;21(1):e7-e10.

10.Gerdes B, Gitei E, Akkermann O, Prasse-Badde J, Schmidt C. Laparoscopic cholecystectomy without visible scar. Combined suprapubic and transumbilical approach: the "Minden cholecystectomy". Endoscopy. 2009;41 Suppl 2:E49-50.

11. Hu H, Zhu JF, Huang AH, Xin Y, Xu AA, Chen B. Covert laparoscopic cholecystectomy:a new minimally invasive technique. Acta Med Okayama. 2011 Oct;65(5):325-8.

12. Leggett $\mathrm{PL}$, Bissell $C D$, Churchman-Winn R. Cosmetic minilaparoscopic cholecystectomy. Surg Endosc. 2001 Oct;15(10):1229-31.

13. Martins MVDCM, Skinovsky J, Coelho DE. Colecistectomia videolaparoscópica por trocater único - SITRACC uma nova opção. Rev. Col. Bras. Cir. 2009;36(2):177-179.

14. Martins MVC, Skinovsky J, Coelho DE, Ramos A, Galvão Neto MP, Rodrigues J, de Carli L, Totti Cavazolla L, Campos J, Thuller F, Brunetti A. Cholecystectomy by single trocar access (SITRACC): the first multicenter study. Surg Innov. 2009 Dec;16(4):313-6.

15. Munive AM.Cirurgia sin cicatriz Em: <http://www.cirugiasincicatriz. com/colecistectomia.html>

16. Spindel IB, Tellez KSM, Munive AM, Garcia JC, Orvañanos FQ.. Minilaparoscopic Approach With Hidden Scars for Usual Laparoscopic Procedures. Poster .Society of American Gastrointestinal and Endoscopic Surgeons Annual Meeting 2012.Disponivel em <http://sages2012.org/minilaparoscopicapproach-with-hidden-scars-for-usual-laparoscopic-procedures/ 